\title{
Photoelectron and ICD electron angular distributions from fixed-in-space neon dimers
}

\author{
T Jahnke ${ }^{1}$, A Czasch ${ }^{1}$, M Schöffler $^{1}$, S Schössler $^{1}$, M Käsz ${ }^{1}$, J Titze $^{1}$, \\ K Kreidi $^{1}$, R E Grisenti ${ }^{1}$, A Staudte ${ }^{2}$, O Jagutzki ${ }^{1}$, L Ph H Schmidt ${ }^{1}$, \\ S K Semenov ${ }^{3}$, N A Cherepkov ${ }^{3}$, H Schmidt-Böcking ${ }^{1}$ and R Dörner ${ }^{1}$ \\ ${ }^{1}$ Institut für Kernphysik, J W Goethe-Universität Frankfurt am Main, Max-von-Laue-Str. 1, \\ D-60438 Frankfurt, Germany \\ ${ }^{2}$ Steacie Institute for Molecular Sciences, 100 Sussex Drive, Ottawa, Canada \\ ${ }^{3}$ State University of Aerospace Instrumentation, 190000 St. Petersburg, Russia \\ E-mail: jahnke@atom.uni-frankfurt.de
}

Received 4 April 2007, in final form 3 May 2007

Published 15 June 2007

Online at stacks.iop.org/JPhysB/40/2597

\begin{abstract}
We report on molecular frame angular distributions of 2s photoelectrons and electrons emitted by interatomic Coulombic decay from neon dimers. We found that the measured angular distribution of the photoelectron strongly depends on the environment of the cluster. The experimental results are in excellent agreement with frozen core Hartree-Fock calculations. The ICD electrons show slight variations in their angular distribution for different kinetic energies.
\end{abstract}

(Some figures in this article are in colour only in the electronic version)

\section{Introduction}

In 1997, Cederbaum and co-workers predicted a strong effect of the chemical environment on the decay of excited atoms and molecules, which they termed 'interatomic Coulombic decay' (ICD) [1]. Their calculations showed that if an excited ion has a close neighbour, like e.g. in van der Waals bonded rare gas clusters or hydrogen bonded systems, it can relax by transferring its excitation energy to this neighbour, causing that neighbour to emit a low energetic electron. ICD occurs on a typical time scale of the Auger decay process (femto-seconds), which is much faster than typical radiative decays $[2,3]$. ICD becomes dominant in all cases where Auger decay at the excited atom itself is blocked due to lack of energy. ICD is fundamentally different from Auger decay because the atom that is excited is not the one that later on emits the electron. 
Apart from opening ICD channels, the environment of an atom can have a second important effect. It can strongly influence the angular distributions of emitted electrons because the electrons can be scattered at neighbouring atoms. The final electron emission pattern is a coherent superposition of all direct and scattered pathways. For covalent bonded molecules such multiple scattering effects have been investigated in detail in recent years in electron/fragment-ion coincidence experiments in the gas phase [4, 29]. On solid surfaces photoelectron diffraction has become a major method for structure analysis [30, 31]. It can even be used for obtaining stereoscopic images of the top surface layers [32] if circularly polarized light is used (see also [17] for studies employing circular light in the gas phase). For van der Waals clusters, so far, only in one pioneering experiment multiple scattering has been reported for argon dimers [33]. In this study, however, no discrepancy between the angular distribution of the monomer and the dimer was found. So, up to now the proof of the influence of the cluster environment on the electron angular distribution has been missing. In this paper we present experimental and theoretical results of such angular distributions of photoelectrons from $\mathrm{Ne}(2 \mathrm{~s})$-photo ionization in a neon dimer clearly showing a strong influence of the neighbour atom.

So far unexplored is the interplay of both environmental effects: ICD and multiple scattering. The ICD electrons can also experience multiple scattering at the cluster environment. So far no theoretical prediction on the angular distributions of ICD electrons is available. Here we present first experimental results on this issue.

The showcase example, which we also have chosen in our study is ICD following 2s-photoionization [34] of neon dimers $\left(\mathrm{Ne}_{2}\right)$. While ICD has first been observed in large clusters [35], the use of dimers yields two advantages: first, dimers allow for a clean identification of the ICD process by detecting the ICD electron in triple coincidence with the vector momenta of the two ionic fragments $[34,36]$. Second, dimers allow for a very detailed theoretical treatment [37]. In a first step (figure 1(a)) the photoelectron is emitted from the $2 \mathrm{~s}$ shell of the $\mathrm{Ne}_{2}$ cluster leaving a $\mathrm{Ne}_{2}^{+}$in either its $\mathrm{g}$ or $\mathrm{u}$ state behind (upper potential curve in figure $1(\mathrm{~d})$ ). The $2 \mathrm{~s}$ hole is filled by a $2 \mathrm{p}$ electron of the same atom. The energy is transferred to the neighbouring atom where the ICD electron is emitted (figure 1(b)). A detailed discussion of the responsible mechanism for ICD being 'energy transfer' or 'electron exchange' can be found in [38]. The IC decay leads from either of the two $\mathrm{Ne}_{2}^{+}\left(2 \mathrm{~s}^{-1}\right)$ curves to the manifold of repulsive $\mathrm{Ne}^{+} / \mathrm{Ne}^{+}$potential energy surfaces in figure 1(d), thus-in a last step - the dimer fragments in a Coulomb explosion.

Due to energy conservation the available total energy is given by the difference of the potential energy of a singly ionized $\mathrm{Ne}\left(2 \mathrm{~s}^{-1}\right)$ atom and two times the ionization potential of a $\mathrm{Ne}(2 \mathrm{p})$-electron by $48.5 \mathrm{eV}-2 \times 21.6 \mathrm{eV}=5.3 \mathrm{eV}$ [39]. The total energy is shared between the ICD electron and the two $\mathrm{Ne}^{+}$ions. Furthermore, according to the law of momentum conservation both $\mathrm{Ne}^{+}$ions have to be emitted back-to-back with almost equal momentum (figure 1(c)) during the Coulomb explosion. These two features-back-to-back emission and a sum energy of the ions and the ICD electron of $5.3 \mathrm{eV}$-provide the fingerprint of the ICD process in this triple coincidence measurement. This fingerprint unambiguously separates the ICD electron from all other low energy electrons, which is essential for measuring its angular distribution. Furthermore, the Coulomb explosion occurs on a much smaller time scale compared to the relatively slow rotation of the dimer. This means that the measured directions of the ionic fragments coincide with the orientation of the dimer at the moment of electron emission. Measuring the directions (i.e. momenta) of the electron and of the ions in coincidence, therefore, allows for a transformation of the electron momenta into the dimer's frame of reference resulting in the molecular frame (or better 'dimer frame') angular distributions that are presented here. 
(a)

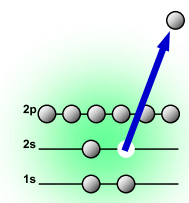

is - $-0-0-0$

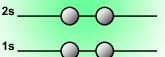

(b)

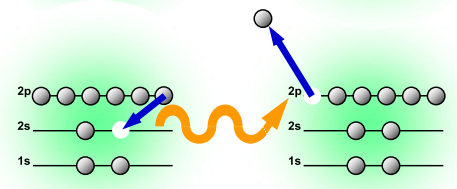

(c)
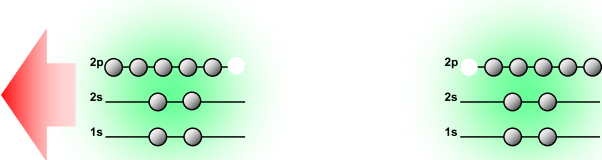

is $-\mathrm{O}-\mathrm{O}$
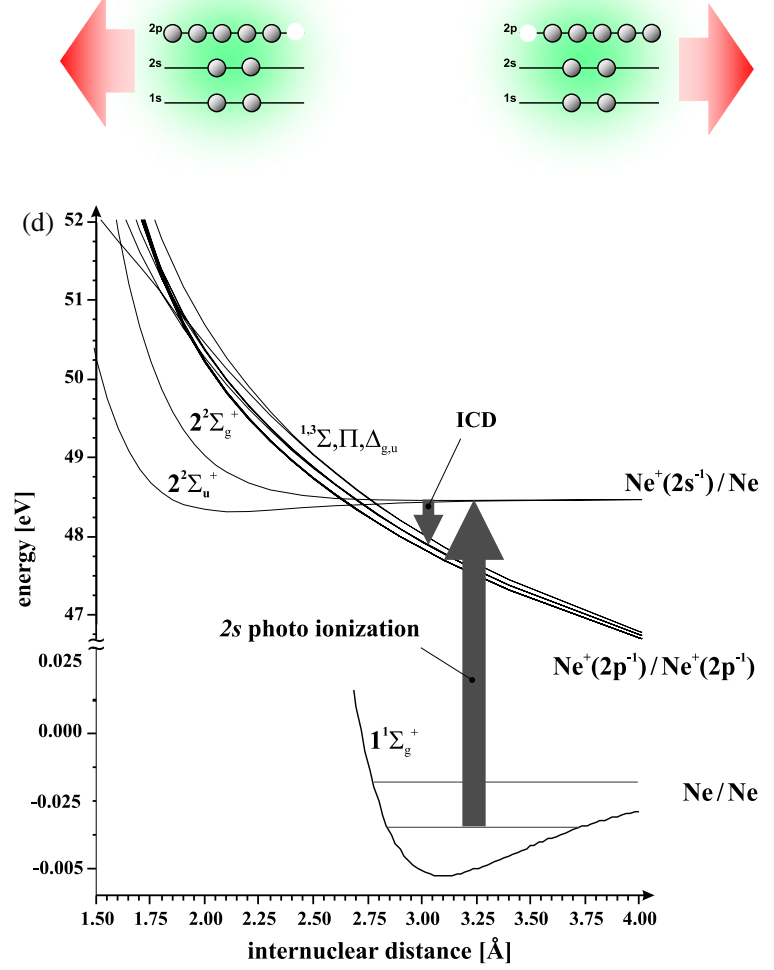

Figure 1. The 2 s photoemission (a) is followed by interatomic Coulombic decay (b): energy is transferred from one atom to its neighbour leading to the emission of an ICD electron. As a final step of the process back-to-back emission of the two $\mathrm{Ne}^{+}$ions occurs as the dimer fragments in a Coulomb explosion (c). (d) Potential energy surfaces relevant for the steps explained above (adapted from [40-42]).

\section{Experiment}

The experiment was performed at the beamline U125/1 PGM of the Berlin electron synchrotron BESSY in single-bunch operation using the COLTRIMS (cold target recoil ion momentum spectroscopy) technique [43-45]. The monochromatic, linearly polarized light was focused onto a supersonic gas jet which contained the neon dimers. The gas jet was produced by expanding neon gas at a driving pressure of 24 bar through a $30 \mu \mathrm{m}$ diameter 


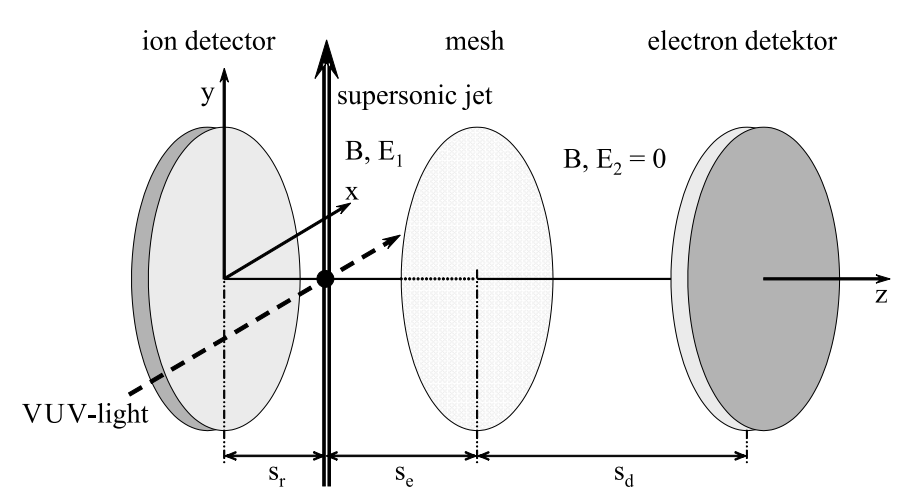

Figure 2. Schematic view of the employed COLTRIMS analyser. Ions are detected on the left, electrons on the right side.

pinhole into a vacuum chamber which was pumped by a $2200 \mathrm{l} / \mathrm{s}$ turbo molecular pump. After passing through a skimmer $(0.3 \mathrm{~mm}$ in diameter, located $12 \mathrm{~mm}$ above the nozzle $)$ the gas jet entered the interaction chamber. We determined the dimer-to-monomer ratio to be $0.7 \%$ using ion time-of-flight spectroscopy after ionization of the $2 \mathrm{p}$ shell at a photon energy of $25 \mathrm{eV}$. The ions and electrons created in the intersection region of photon- and gas beam (located $102 \mathrm{~mm}$ above the nozzle, $0.7 \mathrm{~mm} \times 0.7 \mathrm{~mm} \times 2 \mathrm{~mm})$ were guided by a parallel electric $\left(5.5 \mathrm{~V} \mathrm{~cm}^{-1}\right)$ and magnetic (7.2 Gauss) field towards two position-sensitive multi-channel plate detectors (80 $\mathrm{mm}$ in diameter) equipped with delayline readout [46] (figure 2). The ion detector was placed at a distance of $38.5 \mathrm{~mm}$ from the interaction region. No drift region was used for the ion arm of the spectrometer. The electron arm consisted of a $66 \mathrm{~mm}$ acceleration region and a field-free drift region of $120 \mathrm{~mm}$ in length which were separated by a fine mesh $(0.2 \mathrm{~mm}$ cell width). This spectrometer geometry yielded $4 \pi$ collection efficiency for ions up to an energy of $4 \mathrm{eV}$ and for electrons up to $13 \mathrm{eV}$.

The count rate in the experiment was set to $32 \mathrm{kHz}$ electrons and $10 \mathrm{kHz}$ ions by opening the monochromator slits accordingly. These single-count rates yielded a final rate of about $0.1 \mathrm{~Hz}$ of fully analysable 3-particle coincidence events. The unique fingerprint of ICD in the three-particle phase space allowed for a nearly perfect separation of the real events from the background (see figure 4).

The resolution of the momentum of the electron was mathematically improved in the offline analysis by calculating the exact origin of the reaction using the centre-of-mass position of the detected ion pair for each individual event in the offline analysis as described in [45]. To verify the experimentally achieved angular resolution of the combined ion and electron detection we measured $\mathrm{N}_{2} 1 \mathrm{~s}$ photo ionization during the same beamtime without changing the spectrometer's settings. Figure 3 shows the obtained molecular frame electron angular distribution for the $\mathrm{N}_{2}$ molecule being aligned in parallel (a) and perpendicularly to the polarization axis of the light (b). The contrast at the minima shows that the overall angular resolution of the system is better than $5^{\circ}$ FWHM.

\section{Energy distributions of electrons and ions}

In figure 4 the energy correlation of the measured kinetic energies of the electron and ions (kinetic energy release (KER)) of the $\mathrm{Ne}^{+}$pair is shown for three different photon energies . All photon energies are well below the Ne atom's double ionization threshold. Thus, already 

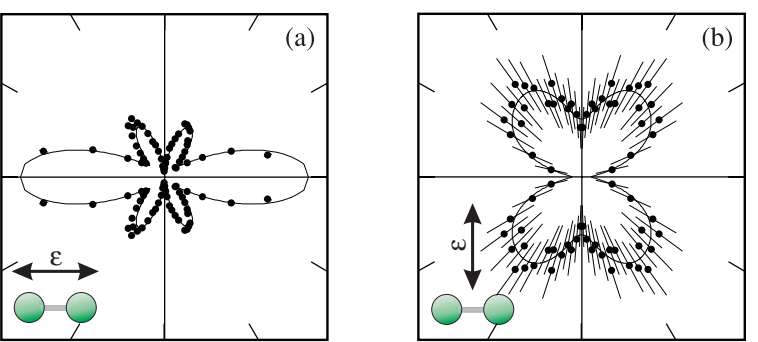

Figure 3. Test measurement of $\mathrm{N}_{2} \mathrm{~K}$-shell photoemission at $h v=420 \mathrm{eV}(11 \mathrm{eV}$ photo electron energy) measured with the same COLTRIMS setup during the same beamtime as the ICD results reported below. The full line is a fit of Legendre polynomials up to $l=5$ to guide the eye. The angular resolution can be estimated from the sharpness of the minima to be better than $5^{\circ} \mathrm{FWHM}$.
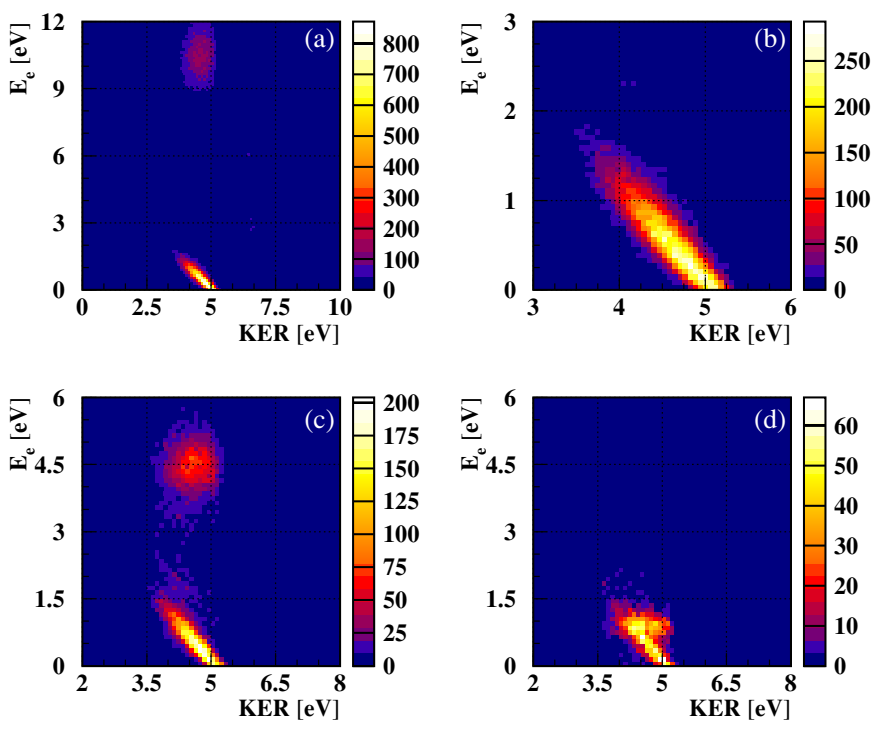

Figure 4. (a) Measured sum kinetic energy of the ions in dependence of the kinetic energy of the electron for a photon energy of $h v=58.8 \mathrm{eV}$. The photo electron peak is located at an electron energy of $11 \mathrm{eV}$, the diagonal feature at an overall sum energy of $5.3 \mathrm{eV}$ clearly shows the existence of ICD electrons. (b) Expanded view of (a) into the region of ICD electrons. (c), (d) Same distribution as in (a) measured at a photon energy of $h v=52.8 \mathrm{eV}$ (c) and $h v=$ $49.3 \mathrm{eV}(\mathrm{d})$.

from simple energy considerations, it becomes clear that the two electrons have to emerge from different atomic sites within the cluster. For our analysis we selected events where the two measured ions are emitted back-to-back. At all three photon energies a diagonal at a sum energy of $5.3 \mathrm{eV}$ clearly identifies the ICD electrons well separated from background. The photo electron can be seen at energies of $11 \mathrm{eV}, 5 \mathrm{eV}$ and $1 \mathrm{eV}$ electron energy, respectively.

By selecting only events along the diagonal of a sum energy of $5.3 \mathrm{eV}$ we obtain the KER distribution for the case that ICD occurred. Photoemission, however, can lead to $\mathrm{Ne}_{2}^{+}$ions either in the $g$ or $u$ state. While the energy shift of the $g$ and $u$ states of the innershell ionized dimer is only approx. $6 \mathrm{meV}$ at the mean internuclear distance of the dimer's groundstate, the nuclear dynamics during decay on these two energy surfaces is highly different, as the 

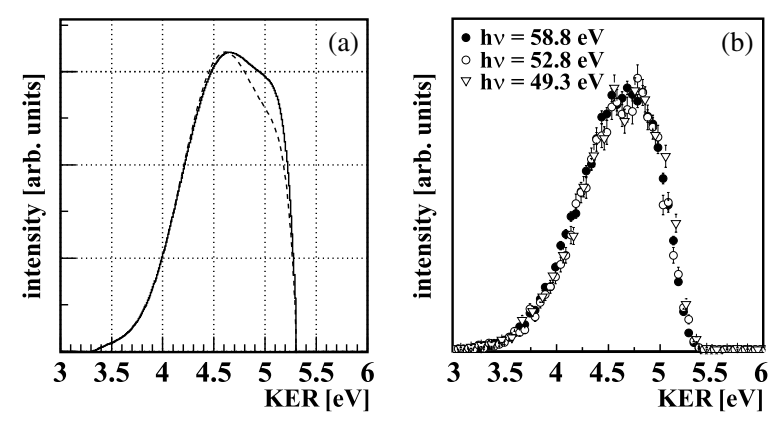

Figure 5. (a) Two simulated KER distributions after ICD (adapted from [37]). Results for a ratio of $\mathrm{g} / \mathrm{u}=50 / 50$ (thin line) and $\mathrm{g} / \mathrm{u}=40 / 60$ (full line) are shown. (b) Measured KER distribution for three photon energies of $h v=58.8 \mathrm{eV}, h v=52.8 \mathrm{eV}$ and $h v=49.3 \mathrm{eV}$.

potential energy surfaces of those two curves show different slopes [41, 42]. In addition to that, the potential of u-symmetry supports a total of 11 vibrational states, while only one stable vibrational level is present for the case of $g$ symmetry. The KER is determined by the internuclear distance at which ICD takes place. Since the ICD lifetime is such that the nuclear wavepacket on the $\mathrm{Ne}_{2}^{+}$curves can evolve before the decay, the distribution of the KER reflects directly the ICD decay time, which itself depends on the internuclear distance, and the nuclear dynamics. For this reason the calculated KER distributions for the $\mathrm{g}$ and $\mathrm{u}$ states show significant differences [37]. In the present case, it is the photoionization cross section which determines the ratio of $\mathrm{g} / \mathrm{u}$ contributions to the final state of the ion. Without any multiple scattering effects one would expect an equal population of $g$ and $u$. In the presence of a shape resonance this can be different as the ratio can then be strongly dependent on the photon energy as such a shape resonance might only occur for one of the two ionization channels (see, e.g., [7] for the case of $\mathrm{N}_{2}$ ). Figure 5(b) shows the measured KER distribution for three different photon energies. Within statistical errors all three distributions coincide. In order to give an impression about what deviations might be expected as the ratio of $\mathrm{g} / \mathrm{u}$ changes, calculated distributions for a ratio of $\mathrm{g} / \mathrm{u}=50 / 50$ and $\mathrm{g} / \mathrm{u}=40 / 60$ (adapted from [37]) are shown in figure $5(\mathrm{a})$. The experimental distribution is most accurately obtained when adding equal contributions from the $\mathrm{g}$ and the $\mathrm{u}$ state, as done in [37, 34]. The close similarity of the experimental KER distribution for the three photon energies suggests that the $g / u$ ratio is nearly constant in the examined range of photon energies.

\section{Angular distributions of photoelectrons}

In a pioneering experiment on the electron angular distribution from fixed-in-space clusters [33], no clear evidence of the influence of the cluster environment on the angular emission pattern was found. In the present case we investigate photoelectrons ejected from the $2 \mathrm{~s}$ shell, thus for the monomer one finds a distribution according to a $\beta$-parameter of $\beta=2-$ a pure dipole distribution. Our results for the dimer at $h v=58.8 \mathrm{eV}(11 \mathrm{eV}$ electron energy) are shown in figure 6. A clear deviation from a dipolar pattern and a dependence on the orientation of the dimer axis with respect to the polarization vector of the light is visible. As in the case of covalent bonded molecules this results from multiple scattering of the electron wave in the two-centre potential. During the scattering process the electron can exchange angular momentum with the cluster ion allowing for angular momentum components beyond $l=1$ in the photo electron wave. The full line in figure 6 shows a fit of Legendre 

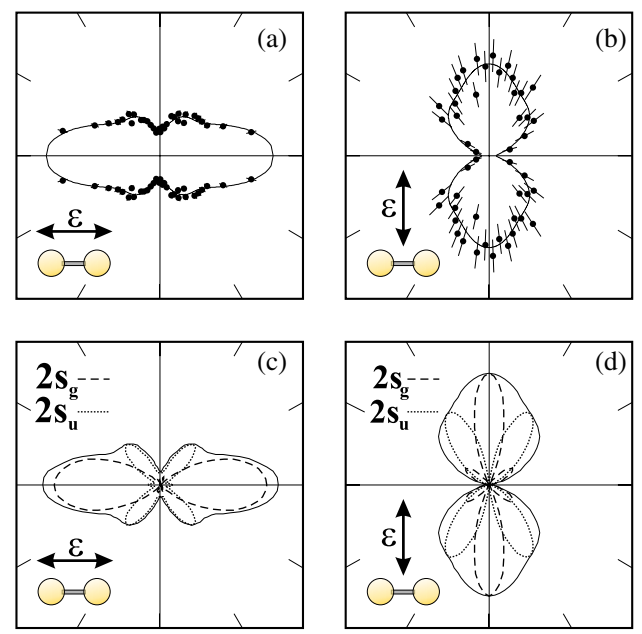

Figure 6. Upper panels: angular distribution of the 2 s photo electron at an electron energy of $11 \mathrm{eV}$. The dimer is oriented horizontally while the polarization vector of the light is oriented (a) in parallel and (b) perpendicularly as shown by the small icons. Lower panels: corresponding results from the FCHF calculation. The dashed line shows the contribution of the ionization to the g state, the dotted line that for the photo ionized state being of u symmetry.

polynomials up to $l=5$ to guide the eye. The experimental results are in excellent agreement with calculations performed in the frozen core Hartree-Fock approximation (FCHF) (see figures $6(\mathrm{c})$ and $(\mathrm{d})$ ). The calculations have been performed using the computer code developed earlier for diatomic molecules in [48]. The fixed molecule photoelectron angular distributions have been found from the equations presented in [8, 17]. In brief, the ground-state HartreeFock wavefunctions of the $\mathrm{Ne}_{2}$ dimer were computed in the prolate spheroidal coordinates using the partial wave expansion up to $l=24$ for the ground state and up to $l=5$ in the continuum. The photoelectron wavefunctions were calculated in the frozen field of the singly charged ion with the hole either in the $2 \sigma_{\mathrm{g}}$ or in the $2 \sigma_{\mathrm{u}}$ shell. That allowed calculating the angular distributions separately for the $2 \sigma_{\mathrm{g}}$ and the $2 \sigma_{\mathrm{u}}$ shell. The corresponding partial angular distributions are also shown in figures $6(\mathrm{c})$ and (d). They clearly visualize how the contributions of the $\mathrm{g}$ and $\mathrm{u}$ shells form the shapes of the experimentally observed angular distributions. The difference between the shapes of the partial angular distributions for the $g$ and $u$ shells is connected to the parity of the photoelectron wavefunction. Namely, only odd partial waves contribute to the ionization of the $2 \sigma_{\mathrm{g}}$ state (according to the FCHF calculations mainly with $l=1$ and 3 ), and only even partial waves (mainly with $l=0,2,4$ ) to the $2 \sigma_{\mathrm{u}}$ state.

\section{Angular distribution of ICD electrons}

To the best of present knowledge the photoemission and the IC decay can be described in a two-step model [49], where photoemission and ICD are treated independently. This implies, in particular, that the ICD angular distribution in the body fixed frame should be independent of the polarization direction. The latter was under dispute for the case of Auger decay in a diatomic molecule [51], but has been re-established today [52]. Consequently, the only relevant axis for the ICD electron angular distribution is the dimer axis and the emission pattern is supposed to be isotropic with respect to the polarization vector of the light. Within our systematical errors this is in agreement with our observations. In figure 7 we show the 

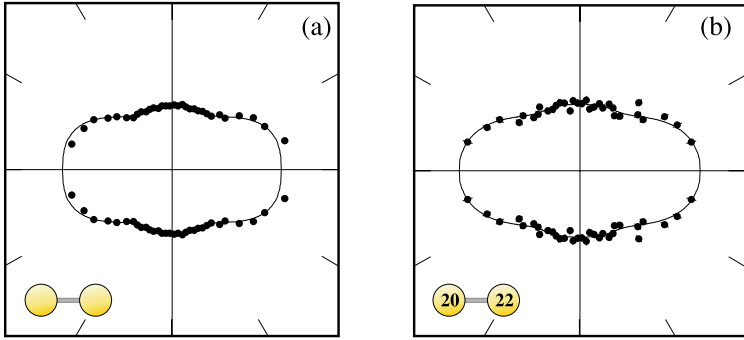

Figure 7. Angular distribution of the ICD electron in the dimer frame. The dimer is oriented horizontally. (a) Emission pattern for a non-isotopic dimer, (b) corresponding distribution for a dimer consisting of a ${ }^{20} \mathrm{Ne}$ and a ${ }^{22} \mathrm{Ne}$ atom. The neon isotope is located at the right, as shown by the small icon.
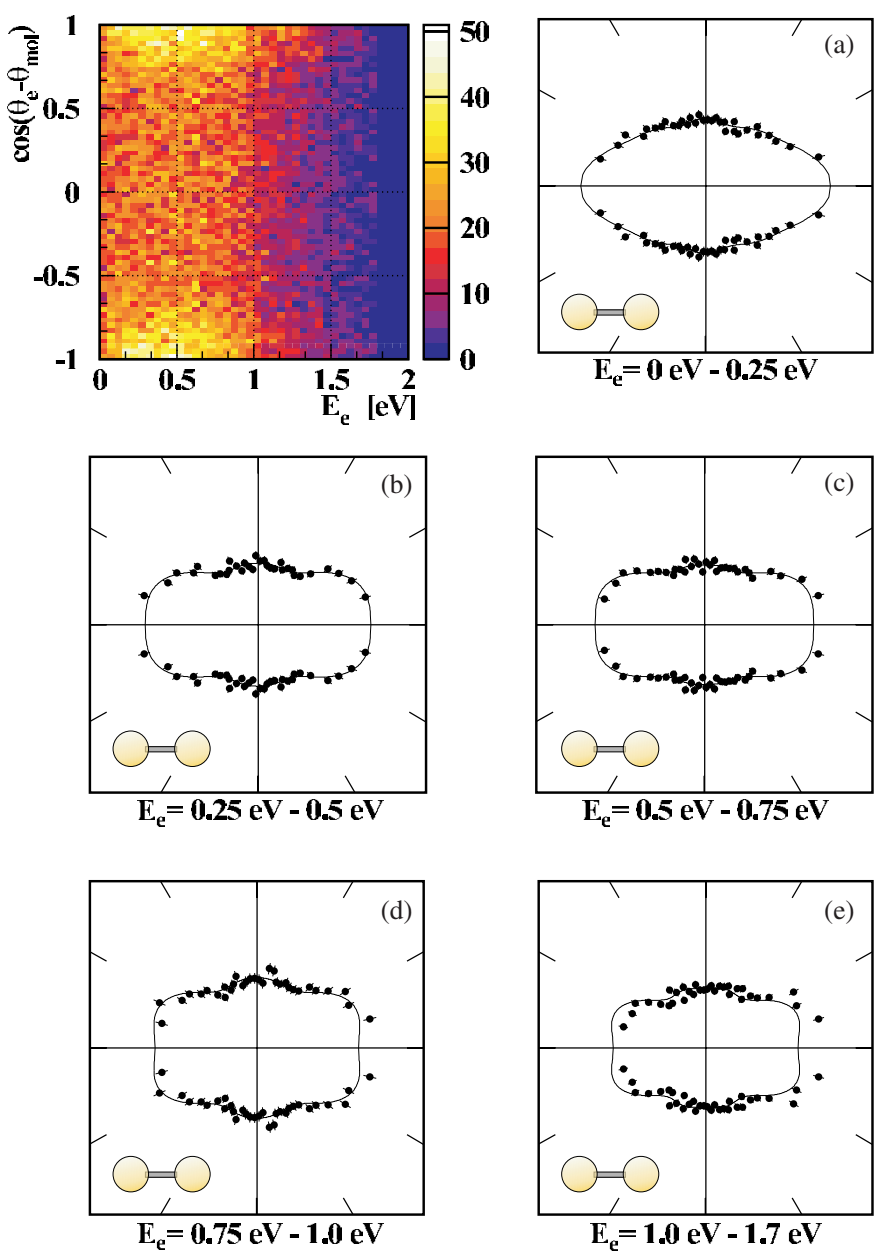

Figure 8. Angular distribution of the ICD electron in the dimer frame for different kinetic energies of the ICD electron. Top left: an overview showing the angular distribution along the $y$-axis and the ICD electron's kinetic energy along the $x$-axis. Parts (a)-(e) display the angular distribution for different slices in the kinetic energy. The full line is a fit with Legendre polynomials up to $l=5$ to guide the eye. 
angular distribution of the ICD electron with respect to the dimer axis and integrated over all measured ICD energies. That distribution is clearly non-isotropic. The ICD electron can emerge from any of the $\mathrm{p}$ subshells of the Ne atom. As in the case of Auger decay, the ICD matrix element contains a factor $1 /\left(r_{1}-r_{2}\right)$ where-in contrast to Auger decay- $r_{1}$ is the coordinate of the electron filling the $2 \mathrm{~s}$ hole on one neon atom and $r_{2}$ is the coordinate of the ICD electron emerging from the other neon atom [50]. Therefore one can expect an enhanced ICD rate for that $\mathrm{p}$ orbital pointing towards the atomic neighbour, i.e. along the dimer axis. This is a likely reason for the preferred emission along the axis which we find.

In figure 7(b) the ICD angular distribution is shown for a isotope-mixed dimer $\left({ }^{20} \mathrm{Ne}{ }^{22} \mathrm{Ne}\right)$. The ${ }^{20} \mathrm{Ne}^{+}$is fixed to the left. However, we do not find any isotope dependence of the angular distribution and no asymmetry. Such isotope effects have been reported previously for photoionization of nitrogen [53].

The ICD electrons will also suffer multiple scattering, which will further shape the angular distribution. It is obvious that a multiple scattering effect is supposed to depend on the kinetic energy of the scattered particle. Figure 8 shows the dimer frame angular distribution of the ICD electron for different kinetic energies. Only minor energy dependence is visible.

\section{Conclusion}

In conclusion, we have reported a pronounced modification of the monomer photoelectron angular distribution by the neighbouring cluster atom in a neon dimer. The effect is in excellent agreement with the FCHF calculations. We clearly identify electrons from interatomic Coulombic decay following 2 s ionization. For these ICD electrons we report the first angular distributions posing a new challenge for the further development of theory of ICD.

\section{Acknowledgments}

The experimental work was supported by the Berliner Elektronenspeicherring für Synchrotronstrahlung (BESSY). We are grateful for discussions with U Hergenhahn, K Ueda, L Cederbaum, S Scheit and V Averbukh. We acknowledge advice on the cluster preparation from Th Möller. We would like to express our thanks to U Hergenhahn and G Reichert for support during the beamtime. SKS and NAC acknowledge the financial support from DFG and INTAS.

\section{References}

[1] Cederbaum L S, Zobeley J and Tarantelli F 1997 Phys. Rev. Lett. 794778

[2] Santra R, Zobeley J and Cederbaum L S 2001 Phys. Rev. B 64245104

[3] Öhrwall G et al 2004 Phys. Rev. Lett. 93203401

[4] Shigemasa E, Adachi J, Oura M and Yagishita A 1995 Phys. Rev. Lett. 74359

[5] Shigemasa E, Adachi J, Soejima K, Watanabe N, Yagishita A and Cherepkov N A 1998 Phys. Rev. Lett. 801622

[6] Watanabe N, Adachi J, Soejima K, Shigemasa E and Yagishita A 1997 Phys. Rev. Lett. 784910

[7] Cherepkov N A, Semenov S K, Hikosaka Y, Ito K, Motoki S and Yagishita A 2000 Phys. Rev. Lett. 84250

[8] Cherepkov N A, Raseev G, Adachi J, Hikosaka Y, Ito K, Motoki S, Sano M, Soejima K and Yagishita A 2000 J. Phys.: Condens. Matter 334213

[9] Ito K, Adachi J, Hall R, Motoki S, Shigemasa E, Soejima K and Yagishita A 2000 J. Phys.: Condens. Matter 33572

[10] Ito K, Adachi J, Hikosaka Y, Motoki S, Soejima K, Yagishita A, Raseev G and Cherepkov N A 2000 Phys. Rev. Lett. 8546

[11] Motoki S, Adachi J, Ito K, Ishii K, Soejima K, Yagishita A, Semenov S K and Cherepkov N A 2002 Phys. Rev. Lett. 88063003 
[12] Golovin A V, Heiser F, Quayle C J K, Morin P, Simon M, Gessner O, Guyon P M and Becker U 1997 Phys. Rev. Lett. 794554

[13] Heiser F, Geßner O, Viefhaus J, Wieliczek K, Hentges R and Becker U 1997 Phys. Rev. Lett. 792435

[14] Gessner O, Hikosaka Y, Zimmermann B, Hempelmann A, Lucchese R R, Eland J H D, Guyon P M and Becker U 2002 Phys. Rev. 88193002

[15] Landers A L et al 2001 Phys. Rev. Lett. 87013002

[16] Weber T et al 2001 J. Phys. B: At. Mol. Opt. Phys. 343669

[17] Jahnke T et al 2002 Phys. Rev. Lett. 88073002

[18] Jahnke T et al 2004 Phys. Rev. Lett. 93083002

[19] Hikosaka Y and Eland J H D 2000 Phys. Chem. Chem. Phys. 204663

[20] Hikosaka Y and Eland J H D 2000 J. Phys. B: At. Mol. Opt. Phys. 333137

[21] Saito N et al 2003 J. Phys. B: At. Mol. Opt. Phys. 36 L24

[22] Lafosse A, Brenot J C, Guyon P M, Houver J C, Golovin A V, Lebech M, Dowek D, Lin P and Lucchese R R 2002 J. Chem. Phys. 1178368

[23] Lafosse A, Brenot J C, Hoejrup K, Guyon P M, Houver J C, Lebech M and Dowek D 2001 J. Chem. Phys. 1146605

[24] Dörner R et al 1998 Phys. Rev. Lett. 815776

[25] Hikosaka Y and Eland J H D 2002 J. Chem. Phys. 27753

[26] Hikosaka Y, Eland J H D, Watson T M and Powis I 2001 J. Chem. Phys. 1154593

[27] Osipov T, Cocke C L, Prior M H, Landers A, Weber T, Jagutzki O, Schmidt L, Schmidt-Böcking H and Dörner R 2003 Phys. Rev. Lett. 90233002

[28] Weber T et al 2004 Nature 431437

[29] Martin F et al 2007 Science $\mathbf{3 1 5} 629$

[30] Fadley C S 1984 Prog. Surf. Sci. 16245

[31] Xu S H, Keeffe M, Yang Y, Chen C, Yu M, Lapeyre G J, Rotenberg E, Denlinger J and Yates J T 2000 Phys. Rev. Lett. 84939

[32] Daimon H 2001 Phys. Rev. Lett. 862034

[33] De Fanis A et al 2004 J. Phys. B: At. Mol. Opt. Phys. 37 L235-42

[34] Jahnke T et al 2004 Phys. Rev. Lett. 93163401

[35] Marburger S, Kugeler O, Hergenhahn U and Möller T 2003 Phys. Rev. Lett. 93203401

[36] Morishita Y et al 2006 Phys. Rev. Lett. 96243402

[37] Scheit S, Averbukh V, Meyer H-D, Moiseyev N, Santra R, Sommerfeld T, Zobeley J and Cederbaum L S 2004 J. Chem. Phys. 1218393

[38] Averbukh V and Cederbaum L S 2006 Phys. Rev. Lett. 96053401

[39] http://webbook.nist.gov

[40] Schirmer J, Cederbaum L S and Walter O 1983 J. Chem. Phys. 281237

[41] Moiseyev N, Santra R, Zobeley J and Cederbaum L S 2001 J. Chem. Phys. 1147351

[42] Santra R, Zobeley J, Cederbaum L S and Moiseyev N 2000 Phys. Rev. Lett. 854490

[43] Dörner R, Mergel V, Jagutzki O, Spielberger L, Ullrich J, Moshammer R and Schmidt-Böcking H 2000 Phys. Rep. 330 96-192

[44] Ullrich J, Moshammer R, Dorn A, Dörner R, Schmidt L Ph H and Schmidt-Böcking H 2003 Rep. Prog. Phys. 66 1463-545

[45] Jahnke T, Weber Th, Osipov T, Landers A L, Jagutzki O, Schmidt L Ph H, Cocke C L, Prior M H, SchmidtBöcking H and Dörner R 2004 J. Electron. Spectrosc. Relat. Phenom. 73 229-38

[46] See http://www.roentdek.com for details on the detectors

[47] Scheit S, Averbukh V, Meyer H-D, Moiseyev N, Santra R, Sommerfeld T, Zobeley J and Cederbaum L S 2004 J. Chem. Phys. 1218393

[48] Semenov S K, Cherepkov N A, Fecher G H and Schönhense G 2000 Phys. Rev. A 61032704

[49] Kuznetsov V V and Cherepkov N A 1996 J. Electron. Spectrosc. Relat. Phenom. 79437

[50] Santra R and Cederbaum L S 2002 Phys. Rep. 368 1-117

[51] Guillemin R, Shigemasa E, Le Guen K, Ceolin D, Miron C, Leclercq N, Morin P and Simon M 2001 Phys. Rev. Lett. 87203001

[52] Weber The et al 2003 Phys. Rev. Lett. 90153003

[53] Rolles D et al 2005 Nature $\mathbf{7 0 5 9} 711$ 